Special issue of the 3rd International Conference on Computational and Experimental Science and Engineering (ICCESEN 2016)

\title{
OFDM Performance of Ultra Wideband in Wireless Body Area Network Channel
}

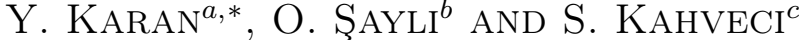 \\ ${ }^{a}$ Recep Tayyip Erdoğan University, Physics Department, Rize, Turkey \\ ${ }^{b}$ Recep Tayyip Erdoğan University, Electrical and Electronics Engineering Department, Rize, Turkey \\ ${ }^{c}$ Karadeniz Teknik University, Electrical and Electronics Engineering Department, Trabzon, Turkey \\ Data transfer for health monitoring can be highly critical because of life dependency transmitted data. IEEE \\ 802.15.6 is a standard for wireless body area network published in 2012 to satisfy the reliability which is required \\ by health monitoring systems. Ultra wideband is one of proposed physical layer technologies specified in the \\ wireless body area network standard which offers high bandwidth, low power, and low interference with other \\ devices. Orthogonal frequency-division multiplexing is commonly used to transmit the data in many wireless \\ communication standards due to its high spectral efficiency and immunity to the inter symbol interference. In this \\ study, bit-error rate performance for orthogonal frequency-division multiplexing with different interpolation based \\ least square channel estimation is given for wireless body area network channel model. OFDM symbol length and \\ pilot length used in OFDM symbol is investigated in bit error rate - signal to noise ratio graphs.
}

DOI: 10.12693/APhysPolA.132.574

PACS/topics: wireless body area network channel, orthogonal frequency-division multiplexing, least square channel estimation, interpolation

\section{Introduction}

Wireless communication network in or around the human body is called body area network. In 2012, IEEE group published IEEE 802.15.6 wireless body area network (WBAN) standard to be used especially in medical applications. The main objective in WBAN is reliability in data transmission. Low power spectral density is another desired specification. One of the introduced physical layer technology is ultra wide band (UWB) wireless communication system [1]. With the high bandwidth of UWB signals, high data rate transmission or lower data rate but more reliable transmission can be achieved [2].

Orthogonal frequency division multiplexing (OFDM) technique has been generally used to meet the requirements of high data rate, reliability and capacity in practical wireless environments, due to its robustness to intersymbol interference (ISI) [3]. Frequency selective channel can be converted into multiple independent flat subchannels by using cyclic prefix (CP) in OFDM systems [4]. However, the low bit error rates (BER) performances of OFDM must be improved by new techniques. The pilot symbols based channel estimators (PSA-CE) is one of the promising techniques for frequency selective channels. PSA-CE with interpolation techniques are widely used for new wireless systems, such as IEEE $802.16 \mathrm{~m}$ worldwide interoperability for microwave access (WiMAX) [5], the third generation partnership project (3GPP) and long-term evolution (LTE) Advanced [6]. Therefore, it can also be applied for WBAN systems [7].

*corresponding author; e-mail: yasin.karan@erdogan.edu.tr
Inserted pilot symbols over subcarriers have disadvantage of a loss in spectral and/or power efficiency. It is desirable to optimize the number of pilot symbols by using better interpolations. The one-dimensional (1D) channel estimations are widely preferred in OFDM based systems to accomplish the trade-off between complexity and accuracy. In this paper, we investigate least square with $1 \mathrm{D}$ interpolation channel estimations for the comb-type pilot arrangement. After that, piecewise commonly used different interpolation techniques are employed to determine the channel frequency responses at data symbols [8].

The rest of this paper is organized as follows. In Sect. 2, basic system model of the OFDM is given. In Sect. 3, WBAN channel models used in this study are given. Simulation results are presented in Sect. 4. Finally, Sect. 5 contains conclusions.

\section{Signal model}

OFDM system block diagram used in UWB-WBAN simulations is shown in Fig. 1. First, the data stream in the transmitter is divided into a block of $N$ complex data symbols presented as

$$
X_{d}=\left[\begin{array}{lllll}
X_{1} & X_{2} & X_{3} & \ldots & X_{N}
\end{array}\right]^{T}
$$

where the symbols are taken from M-QAM constellations where $\mathrm{M}$ is the constellation size and $X_{d}$ shows modulated signal array. Then, $N$-point IFFT is applied on the data to transform into time domain as shown in Eq. (2):

$$
x[n]=\frac{1}{\sqrt{N}} \sum_{k=0}^{N-1} X[k] \mathrm{e}^{\mathrm{j} \frac{2 \pi k n}{N}} .
$$

After IFFT last $N_{g}$ sample whose length chosen longer than expected delay spread of the WBAN channel is added as a cyclic extension (CP) to time domain signal $x[n]$ to avoid inter-symbol interference (ISI) as follows: 


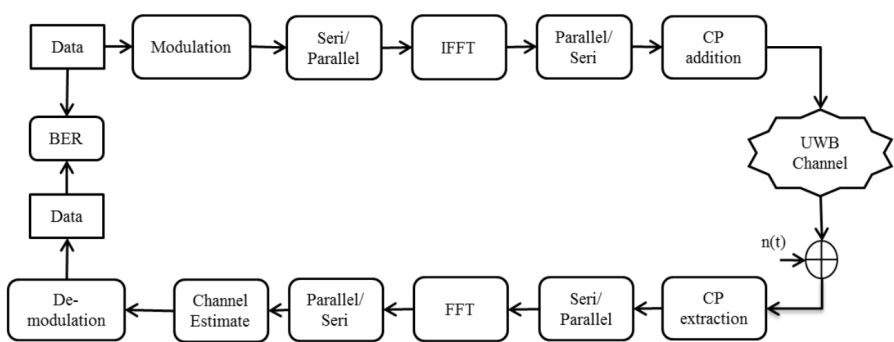

Fig. 1. OFDM block diagram.

$$
\begin{gathered}
\tilde{x}[n]=x\left[N-N_{g}+n\right] \bmod (N), \\
1 \leq n \leq N+N_{g}-1 .
\end{gathered}
$$

The received signal which is deteriorated by the channel, $h[n]$, and additive white Gaussian noise, $w[n]$, is given in Eq. (4):

$$
r[n]=\sum_{i=0}^{L} h[l] x[n-l+w[n]] .
$$

At the receiver, OFDM symbols first passed through the serial to parallel converter. After the signal is parallelized, the $\mathrm{CP}$ is removed from received signal. In final step to get flat fading channel at each subcarrier, the OFDM signal passes through the fast Fourier transform (FFT) operator as shown in Eq. (5):

$$
R[k]=\sum_{i=0}^{L} r[n] \mathrm{e}^{-\mathrm{j} \frac{2 \pi k n}{N}} .
$$

The received signal contains data symbol, channel effects, and AWGN noise. The channel at the pilot subcarriers is estimated with least square zero forcing filter as in Eq. (6) and Eq. (7). The output of the filter is interpolated by nearest, linear, pchip, and spline interpolation methods to estimate the channel at data subcarriers [9]. The interpolation for the pilot data can be realized with different methods. The list of formula and complexity is given in Table I. Figure 2 shows samples of how different interpolation methods work.

$$
\begin{aligned}
R\left[k_{p}\right] & =\rho H\left[k_{p}\right]+w\left[k_{p}\right], \\
\hat{H}\left[k_{p}\right] & =R\left[k_{p}\right] / \rho,
\end{aligned}
$$

where $\hat{H}$ is estimated channel, $\rho$ is pilot symbol. Here, the AWGN noise is neglected.

\section{Channel model}

Four different channel models are used in BAN, based on the location of transmitters. Sensor transmitters and hub can be in-body, on-body, and out of body. IEEE 802.15 group published these channel models in 2010 [10]. In this study, channel model 4 (CM4) which is on-body out of body channel is used. The channel model provides the power delay profile as in Eq. (8):

$$
h(T)=\sum_{l=0}^{L-1}\left(a_{l} \mathrm{e}^{-\mathrm{j} \Phi_{l}} \delta\left(t-t_{l}\right)\right),
$$

where $a_{l}$ is path amplitude, $t_{l}$ is arrival time, $\Phi_{l}$ is phase

\begin{tabular}{|c|c|c|}
\hline Type & Formula & Complexity \\
\hline nearest & nearest point & 1 point is enough, simplest \\
\hline linear & $\mid \begin{array}{l}y=y_{a} \\
\left.y_{a}\right) \frac{x-x_{a}}{x-x_{a}}\end{array}$ & 2 points necessary, simple \\
\hline pchirp & $\begin{array}{l}3 \text { rd degree } \\
\text { polynomials }\end{array}$ & $\begin{array}{l}4 \text { points necessary uses } \\
\text { polynomial. }\end{array}$ \\
\hline spline & $\begin{array}{l}\text { 3rd degree } \\
\text { polynomials }\end{array}$ & $\begin{array}{l}4 \text { points necessary, } \\
\text { smoother than pchip } \\
\text { but the most complex }\end{array}$ \\
\hline
\end{tabular}
for $l$-th path and $L$ is the total number of paths. $\Phi_{l}$ phase
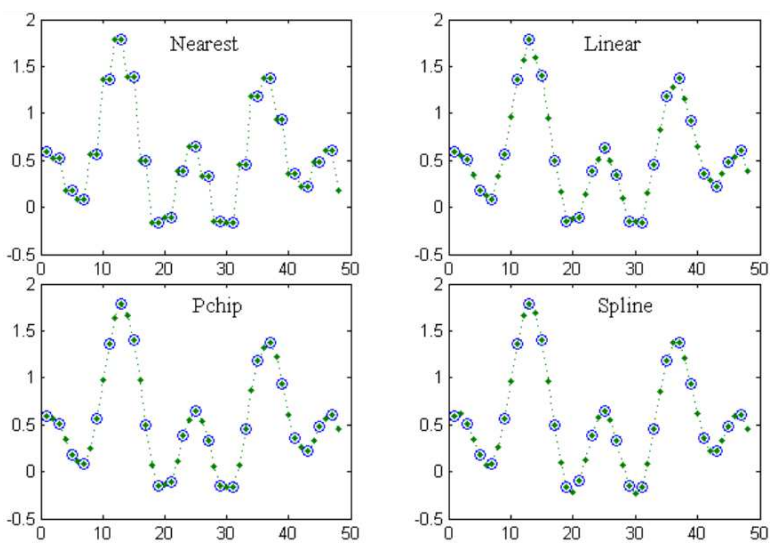

Fig. 2. Different interpolations methods samples.

Interpolation types.

TABLE I

is modelled as uniform disturbed in $[0,2 \pi]$ interval. $a_{l}$ is modelled as in Eq. (9):

$$
10 \log \left|a_{l}\right|^{2}=\left\{\begin{array}{ll}
0, & l=0 \\
\Upsilon_{0}+10 \log \mathrm{e}^{-\frac{t_{l}}{\Gamma}}+S, & l \neq 0
\end{array},\right.
$$

where $S$ is log-normal modeled stochastic term with zero mean, $\sigma_{s}$ standard derivation. $\Gamma$ is exponential distortion constant and $\Upsilon_{0}$ is the Rician factor. Arrival time $t_{l}$ is modeled as the Poisson distribution with $\lambda$ mean time between arrivals as in Eq. (10). The constant are defined in [9] according to model types

$$
p\left(t_{l} \mid t_{l-1}\right)=\lambda \mathrm{e}^{-\lambda\left(t_{l}-t_{l-1}\right)} \text {. }
$$

Figure 3 shows the channel delay profile that was used in the simulations. Channel Model Four with $0^{\circ}$ angle between transmitter and receiver is used. Average multipath number is chosen one hundred.

\section{Simulation results}

Simulations are realized based on interpolation types, subcarrier-pilot numbers and modulation types with BPSK modulation. The results based on interpolation methods are shown in Fig. 4a and b for 512 and 1024 subcarrier and $1 / 2$ pilot-subcarrier ratios, respectively. Nearest, linear, piecewise cubic hermite interpolating polynomial (PCHIRP) and spline methods are used and it is seen that spline method gives better performance than the others. Number of the pilot is crucial for reliable data transmitting. It is seen that when pilot data number is not high, only spline interpolation gives sufficient performance. 


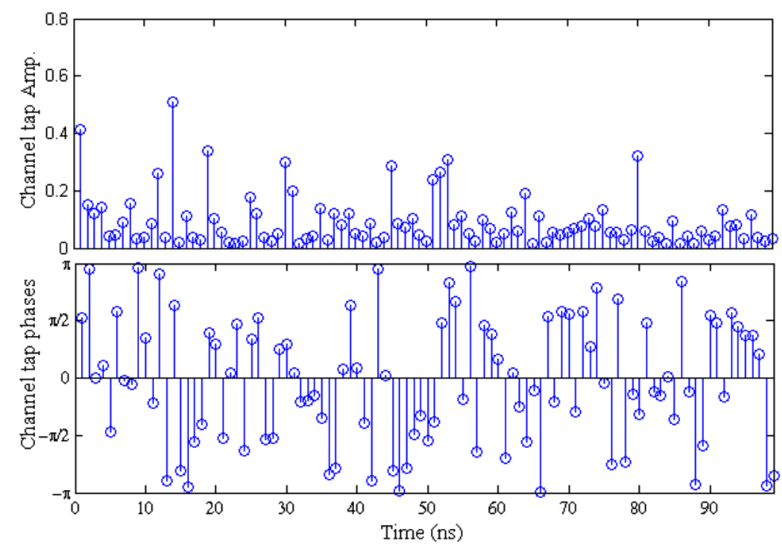

Fig. 3. Channel power delay profile.

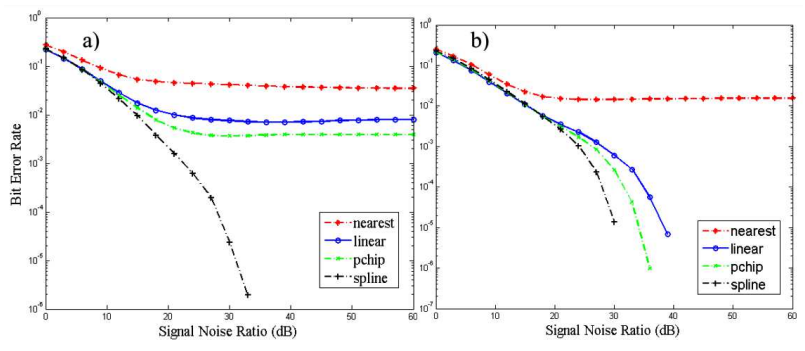

Fig. 4. Performance for different interpolation types, subcarrier and pilot numbers are (a) 512-256, (b)1024512 .

Figure 5a and b shows the performance based on the number of subcarrier-pilot data for linear and spline interpolations, respectively with BPSK modulation. In lower pilot subcarrier number, spline gives better performance than the linear interpolation. When the pilot rate is higher than $1 / 2$, linear interpolation gives better performance and it can be chosen because of lower complexity. Additionally, complexity for interpolation methods can be seen in Table I. The tradeoff is between pilot numbers (carrier number and data rate) and the complexity of interpolation. Also, from Fig. 5b, when the pilot rate is $3 / 4$ of the total subcarriers, the spline method did not improve the performance, because estimation accuracy of the polynomial got worse.

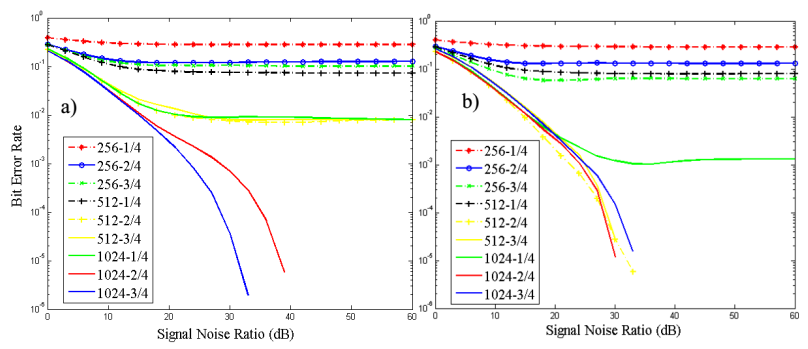

Fig. 5. Performance for subcarrier-pilot number, interpolation is (a) linear, (b) spline.

Figure $6 \mathrm{a}$ and $\mathrm{b}$ shows the performance due to the modulation types 512 and 1024 subcarrier and $1 / 2$ pilotsubcarrier ratio, respectively. Spline is used as interpo-

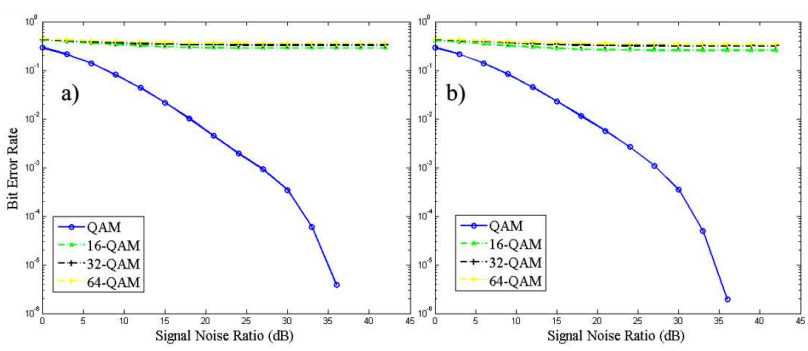

Fig. 6. Performance for different modulations, subcarrier \# is (a) 512, (b) 1024.

lation method. In WBAN channel higher order modulations are not capable for data transmission.

\section{Conclusion}

In this study, performance of interpolation and subcarrier number for OFDM are analysed in WBAN channel. The spline interpolation gives the higher performance in WBAN channel. For the least square estimator, number of the pilot symbol plays critical role for performance, due to the pilot symbol number, performance may go to error floor. Also, different QAM types are searched, and higher than the 4-QAM is not capable for transmission, because the symbols are close to each other and hard to distinguish after the WBAN channel and AWGN.

\section{References}

[1] IEEE Standards Association, IEEE standard for local and metropolitan area networks - Part 15.6: wireless body area networks, IEEE Standard for Information Technology, IEEE, 2012, Vol. 802, p. 1.

[2] Y. Karan, S. Kahveci, in: Proc. IEEE 24th Signal Processing and Communication Application Conf. (SIU), Zonguldak (Turkey), 2016, p. 285.

[3] M. Tören, C. Çiflikli, Acta Phys. Pol. A 130, 417 (2016).

[4] O. Şaylı, H. Doğan, E. Panayırcı, in: Proc. IEEE 24th Signal Processing and Communication Application Conference (SIU), Zonguldak (Turkey), 2016, p. 333.

[5] J.G. Andrews, A. Ghosh, R. Muhamed, Fundamentals of WiMAX: Understanding Broadband Wireless Networking, Pearson Education, 2007.

[6] E. Dahlman, S. Parkvall, J. Skold, 4G: LTE/LTEAdvanced for Mobile Broadband, Academic Press, 2013.

https://www

[7] A. Batra, J. Balakrishnan, A. Dabak, Microwave Engineering Online (28.12.2003).

[8] S. Coleri, M. Ergen, A. Puri, A. Bahai, IEEE Trans. Broadcast. 48, 223 (2002).

[9] J.B. Wang, Y. Jiao, X.Y. Dang, M. Chen, X.X. Xie, L.L. Cao, Optoelectron. Lett. 7, 213 (2011).

[10] K. Yazdandoost, K. Sayrafian-Pour, "TG6 channel model ID: 802.15-08-0780-12-0006", IEEE submission, Nov. 2010. 